\title{
Expression of LKB1 tumor suppressor in non-small cell lung cancer determines sensitivity to 2-deoxyglucose
}

\author{
Landon J. Inge, PhD, Keith D. Coon, PhD, Michael A. Smith, MD, and Ross M. Bremner, MD, PhD
}

\begin{abstract}
Objective: Targeted therapy promises to improve patient outcome in non-small cell lung cancer. Biomarkers can direct targeted therapy toward patients who are most likely to respond, thus optimizing benefit. A novel agent with antineoplastic potential is the glucose analog, 2-deoxyglucose. 2-Deoxyglucose targets tumor cells, owing to their increased glucose uptake, inhibiting cellular metabolism and inducing energetic stress, resulting in decreased cellular viability. The tumor suppressor LKB1 is activated by energetic stress, and cells that lack LKB1 fail to respond and undergo cell death, suggesting that LKB1-null non-small cell lung cancer may have an increased susceptibility to 2-deoxyglucose. Inasmuch as somatic loss of LKB1 is a frequent event in non-small cell lung cancer, LKB1 expression could be used as a biomarker for directing 2-deoxyglucose therapy in patients with this type of cancer.
\end{abstract}

Methods: LKB1-positive and LKB1-negative non-small cell lung cancer cell lines were evaluated for cell viability, markers of apoptosis, and gene expression after 2-deoxyglucose treatment and compared with vehicle control.

Results: LKB1-negative cells treated with 2-deoxyglucose displayed a significant decrease in cell viability compared with LKB1-positive cells. Gene expression profiles of 2-deoxyglucose treated cells revealed changes in apoptotic markers in LKB1-negative cells, correlating with activation of apoptosis. Re-expression of LKB1 prevented 2-deoxyglucose mediated apoptosis, demonstrating the critical role of LKB1 in mediating 2-deoxyglucose toxicity.

Conclusions: LKB1 loss increases susceptibility to 2-deoxyglucose treatment in non-small cell lung cancer lines, even at low doses. Thus, determination of LKB1 status may help direct therapy to those patients most likely to benefit from this novel approach, making it useful in the treatment of patients with non-small cell lung cancer.

Although recent statistics reveal significant increases in survival for many cancers, survival rates for lung cancer have seen little improvement over the past few decades, with overall patient 5 -year survival approximately $15 \% .{ }^{1}$ Currently, curative treatment of non-small cell lung cancer (NSCLC) is restricted to surgical resection for very early disease, with the addition of systemic chemotherapy for patients with later stages of cancer, inasmuch as metastatic recurrence is common. Unfortunately, the response rates for NSCLC to traditional chemotherapeutic agents has been poor, although the more recent attempts at personalizing chemotherapy appear to hold promise for improving outcomes. Certainly, the understanding of epidermal growth factor receptor (EGFR) mutations in some cancers has helped direct application of tyrosine kinase inhibitors toward patients with these distinct mutations. ${ }^{2}$ The success of this

From the Center for Thoracic Disease, Heart and Lung Institute, St Joseph's Hospital and Medical Center, Phoenix, Ariz.

This work was funded by grant from the St Joseph's Foundation (Phoenix, Ariz) for the Heart and Lung Institute Research Initiative.

Received for publication June 11, 2008; revisions received Sept 16, 2008; accepted for publication Nov 20, 2008.

Address for reprints: Ross M. Bremner, MD, PhD, Heart and Lung Institute, St Joseph's Hospital and Medical Center, 500 West Thomas Rd, Suite 500, Phoenix, AZ 85013 (E-mail: ross.bremner@chw.edu).

J Thorac Cardiovasc Surg 2009;137:580-6

$0022-5223 / \$ 36.00$

Copyright (c) 2009 by The American Association for Thoracic Surgery

doi:10.1016/j.jtcvs.2008.11.029 approach has stimulated the idea that certain characteristics of each individual cancer can be exploited to improve outcomes for the disease. Thus, the paradigm in lung cancer is changing toward one of personalized therapy. The LKB1 tumor suppressor gene, which is commonly mutated in NSCLC, offers one such opportunity.

The LKB1 gene was discovered through genetic linkage analysis of the familial disorder, Peutz-Jeghers syndrome ${ }^{3}$ and has since been found to be inactivated in $30 \%$ to $50 \%$ of patients with NSCLC, ${ }^{4-6}$ about twice the prevalence of EGFR mutations found in this disease. Further, convergent in vitro and in vivo studies have led to the realization that loss of LKB1 may be a critical event in NSCLC. ${ }^{6-9}$ LKB1 is a serine-threonine kinase, phosphorylating and regulating 14 different protein kinases. ${ }^{3}$ The biological role of LKB1 regulation of these kinases remains largely unknown except for the adenosine monophosphate (AMP)-activated protein kinase, or AMPK. The primary function of LKB1-AMPK signaling is in the regulation of cellular energy metabolism. Increases in intracellular levels of AMP, caused by hypoxia, ischemia, or other stressors, induce the LKB1-dependent activation of AMPK, allowing AMPK to alter cellular functions and restore adenosine triphosphate levels within the cell. ${ }^{3,9}$ One such process regulated by AMPK during low energy states is cell growth. ${ }^{10}$ Inasmuch as deregulation of cell growth is a critical feature of cancer, this function of LKB1 and AMPK has led to an increased interest in their roles in 


$$
\begin{aligned}
& \text { Abbreviations and Acronyms } \\
& \begin{aligned}
\text { AMP } & =\text { adenosine monophosphate } \\
\text { AMPK } & =\text { AMP-activated protein kinase } \\
2-D G ~ & =2 \text {-deoxyglucose } \\
\text { EGFR } & =\text { epidermal growth factor receptor } \\
\text { KDLKB1 } & =\text { kinase-dead LKB } 1 \\
\text { mTOR } & =\text { mammalian target of rapamycin } \\
\text { NSCLC } & =\text { non-small cell lung cancer } \\
\text { PARP } & =\text { poly (ADP-ribose) polymerase } \\
\text { TBST } & =\text { Tris buffered saline/0.1\% Tween } 20 \\
\text { TSC1/2 } & =\text { tuberous sclerosis complex }
\end{aligned}
\end{aligned}
$$

malignancy. LKB1 and AMPK negatively affect cell growth by inhibition of the protein kinase, mTOR (mammalian target of rapamycin), which functions in increasing cell growth and is commonly deregulated in cancer. ${ }^{11}$ LKB1-AMPK regulation of mTOR occurs via AMPK activation of the tuberous sclerosis complex (TSC1/2) tumor suppressors, which inhibit mTOR activation. ${ }^{10,12}$ Interestingly, stimuli that normally activate LKB1-AMPK fail to result in decreased mTOR activity in LKB1-null cells ${ }^{12-14}$ and can activate apoptosis (Figure 1). These and other findings have established a direct link between the LKB1-AMPK and the mTOR signaling pathways.

During the 1920s, Otto Warburg made the discovery that tumor cells rely on glycolysis, as opposed to normal mitochondrial oxidative phosphorylation, for energy production (the "Warburg effect"). ${ }^{9}$ Recent work in basic and clinical research has improved this basic understanding, demonstrating that tumor cells have uniquely altered metabolisms, displaying increased glucose uptakes, increases in enzymes of the glycolytic pathway, and an increased sensitivity to inhibition of glycolysis. ${ }^{9}$ The increased uptake of glucose and lack of glucose-6-phosphatase activity (which directs glucose exit from the cancer cell) has led to the clinical use of the glucose analog $\left[{ }^{18} \mathrm{~F}\right]$ fluoro-deoxyglucose, in positron emission tomography scanning. Another glucose analog, 2-deoxyglucose (2-DG), inhibits glycolysis and appears to be preferentially toxic to tumor cells. ${ }^{15-17}$ Studies have found that 2-DG has potential in treating cancer and is being investigated currently in phase I trials. It is also being explored as a treatment for epilepsy as a surrogate for the "ketogenic diet," 18 and it appears to be well tolerated in early studies. Fortuitously, and of significant importance, 2-DG is also a specific activator of LKB1-AMPK signaling, suggesting that LKB1-AMPK may be critical in mediating the antitumor effects of 2-DG.

In this study, we examined the response of NSCLC cell lines to the glycolytic inhibitor 2-DG and the relationship of the response to the function of the LKB1 tumor suppressor gene. We hypothesized that loss of LKB1 function

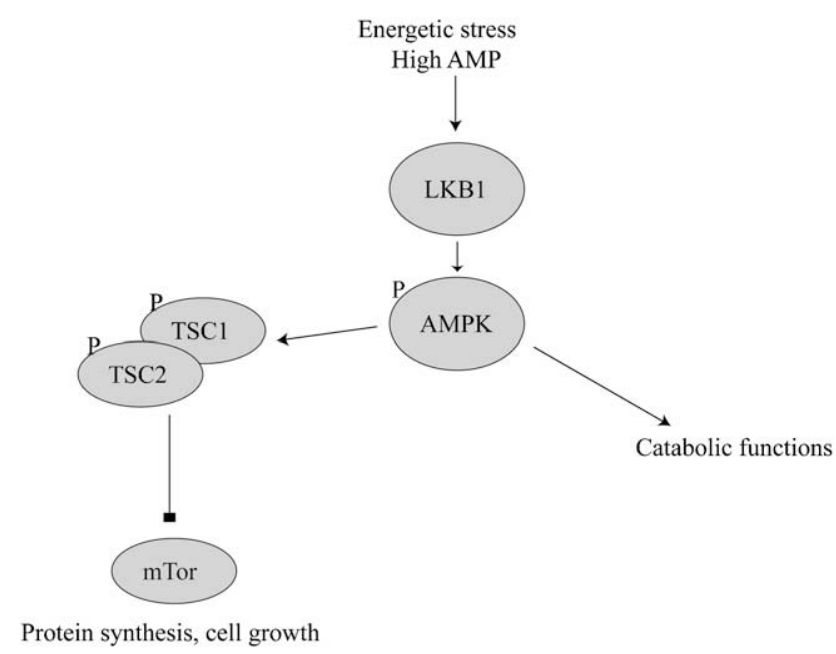

FIGURE 1. LKB1-AMPK signaling and regulation of mammalian target of rapamycin ( $m T O R$ ). TSC, tuberous sclerosis complex; AMPK, adenosine monophosphate kinase.

would render NSCLC cancer cell lines more susceptible to glycolytic stress. If true, this would provide a unique opportunity to identify future patients with NSCLC who would be susceptible to 2-DG therapy in the clinical setting.

\section{METHODS}

\section{Reagents and Cell Culture}

H23, H2009, H2122, and H441 lung adenocarcinoma cell lines were obtained from ATCC (Manassas, Va) and maintained in RPMI 1640 (Invitrogen, Carlsbad, Calif) $/ 10 \%$ fetal bovine serum under standard tissue culture conditions. Antibodies to LKB1, phosphorylated AMPK, AMPK, and cleaved poly (ADP-ribose) polymerase (PARP) were purchased from Cell Signaling Technology (Danvers, Mass). CellTiter Blue and Caspase-Glo 3/7 kits were purchased from Promega (Madison, Wis). 2-DG was purchased from Sigma Chemical Co (St Louis, Mo) and diluted to a 1-mol/L stock in sterile phosphate-buffered saline. The pBABE retroviral constructs containing FLAG-tagged full-length LKB1 and the KDLKB1 originated in Dr Lewis Cantley's laboratory (Harvard Medical School, Boston, Mass) and were obtained from Addgene.org (Cambridge, Mass).

\section{Cell Lysis and Immunoblotting}

Cells were incubated on ice for 30 minutes with a lysis buffer containing $10 \mathrm{mmol} / \mathrm{L}$ Tris- $\mathrm{HCl}, 150 \mathrm{mmol} / \mathrm{L} \mathrm{NaCl}$, and $1 \%$ IGEPAL (Sigma). Immediately before use, a protease inhibitor cocktail (Sigma) and phosphatase cocktails I and II (Sigma) were added to the lysis buffer. Lysates were transferred to tubes and insoluble material was pelleted by centrifugation. Protein concentration was determined by the Bio-Rad DC protein kit (Bio-Rad Laboratories, Hercules, Calif). For immunoblotting, $100 \mu \mathrm{g}$ of total protein lysate was separated by sodium dodecyl-sulfate-polyacrylamide gel electrophoresis and transferred to nitrocellulose. Blots were blocked in 5\% milk/Tris buffered saline/ $0.1 \%$ Tween 20 (TBST) and primary antibodies were diluted in $5 \%$ bovine serum albumin (Sigma)/TBST and incubated overnight at $4^{\circ} \mathrm{C}$. Blots were developed with Amersham ECL Plus (GE Healthcare, Waukesha, Wis) and visualized on a Kodak Image station.

\section{Cell Viability}

Cell lines were plated at a density of 2500 cells per well in 96 well plates. After attachment, 2-DG was diluted in media at indicated concentrations 


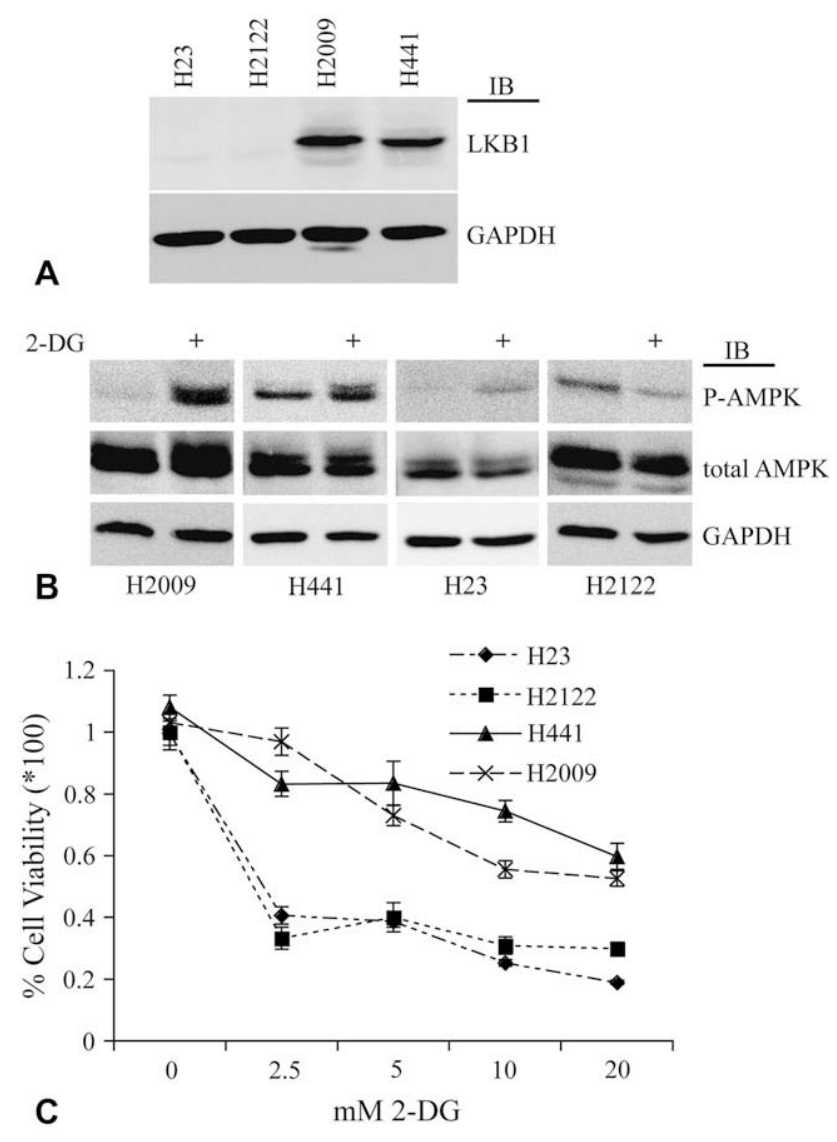

FIGURE 2. Effect of 2-DG in LKB1-null and LKB1-expressing NSCLC cells. A, Immunoblot of LKB1 in H23, H2122, H2009, and H441 NSCLC cell lines. GAPDH was used as a loading control. B, Induction of AMPK phosphorylation by 2-DG. LKB1-positive (H2009, H441) and LKB1-negative (H23, H2122) cell lines were treated with $20 \mathrm{mmol} / \mathrm{L}$ of 2-DG for 1 hour. Protein lysates were immunoblotted with an antibody specific to phosphorylated Thr172 AMPK. Note 2-DG induces phosphorylation at Thr172 only in LKB1-expressing cells. Both AMPK and GAPDH were used as loading controls. Blot is representative of two independent experiments. C, LKB1 null NSCLC cell are more sensitive to 2-DG. NSCLC cell lines were treated for 48 hours with indicated concentrations of 2-DG. Bars represent standard error. GAPDH, Glyceraldehyde-3-phosphate dehydrogenase; 2-DG, 2-deoxyglucose; $A M P K$, adenosine monophosphate activated protein kinase; $P-A M P K$, adenosine monophospahte kinase phosphorylation.

and added to wells. Treatment was carried out for 48 hours in standard cell culture conditions, before cell viability was assessed by the CellTiter Blue kit (Promega) according to manufacturer instructions and read on a Beckman Coulter DTX 880 . The percentage of viable cells was determined by normalizing treated samples to vehicle (phosphate-buffered saline).

\section{Caspase 3/7 Activity}

Cell lines were plated at a density of 5000 cells per well in 96 well plates. After attachment, cells were treated with either 2-DG or vehicle for 24 hours. Activity of caspases 3 and 7 was determined by a Caspase-Glo 3/7 kit (Promega) according to manufacturer instructions and scanned with a Beckman Coulter DTX 880 plate reader (Beckman Coulter, Fullerton, Calif). Relative light units from treated samples were normalized to vehicle controls to generate fold activity of caspases 3 and 7 .

\section{Gene Expression Profiling}

Total RNA was extracted from cells using TRIzol (Invitrogen) followed by RNeasy Mini Kit purification (Qiagen, Venlo, Netherlands) according to manufacturer specifications. Spectrophotometric analysis was performed on a NanoDrop ND-1000 (Thermo Scientific, Waltham, Mass) and RNA quality was assessed on a BioAnalyzer 2100 (Agilent, Santa Clara, Calif) using strict RNA integrity QC cutoffs. Next, $500 \mathrm{ng}$ of RNA was labeled and hybridized (in duplicate) to Agilent $4 \times 44 \mathrm{~K}$ Whole Human Genome OneColor Oligo Microarrays according to manufacturer specifications. Slides were scanned using the Agilent Microarray Scanner (model G2505B) and processed with Agilent's Feature Extraction software (v. 9.5.1). Comparative and statistical analyses for gene expression profiles were carried out with GeneSpring 7.3 software (Silicon Genetics, Inc, Redwood City, Calif). Apoptosis-related genes of interest were identified by key word search ("apoptosis") followed by unsupervised hierarchical clustering. Significance values were determined by comparing expression of all LKB1-positive samples (grouped) to all LKB1-negative samples (grouped) for each gene using the Student $t$ test and a $P<.05$ cutoff after multiple testing correction using the false discovery rate method.

\section{RESULTS}

To investigate the role of LKB1 in the effectiveness of 2DG treatment, we screened four NSCLC cell lines (H23, H2122, and H441, H2009) by immunoblot for LKB1 expression (Figure 2, A). Exposure of cells to $20 \mathrm{mmol} / \mathrm{L}$ of 2-DG induced LKB1-dependent activation of AMPK, confirming published data (Figure 2, B). Inasmuch as LKB1AMPK signaling can affect cell growth or cell number, we compared the number of viable LKB1-null and LKB1-positive cells after treatment with 2-DG for a range of concentrations over time. After 48 hours of treatment, both LKB1-positive and LKB1-negative cells showed a significant decrease in cell viability at high doses $(20 \mathrm{mmol} / \mathrm{L})$ of 2-DG (Figure 2, C), thus confirming the ability of 2-DG to limit cell number. However, LKB1-null cells showed a significant decrease in cell viability for all doses tested, even at the lowest dose $(2.5 \mathrm{mmol} / \mathrm{L})$ compared with LKB1-positive cells $\left(P=5.73 \cdot 10^{-13}\right)$. These findings suggested that LKB1-null NSCLC cells are significantly more sensitive to treatment with 2-DG than LKB1-positive cells and that 2-DG might induce a different response in LKB1null NSCLC cells.

Activation of energetic stress by glucose starvation results in the induction of apoptosis or programmed cell death in both LKB1-null NSCLC cells and LKB1-null mouse embryonic fibroblasts. ${ }^{12-14}$ Inasmuch as 2-DG had a more significant effect on cell viability in LKB1-null but not LKB1-positive NSCLC cell lines, we hypothesized that 2-DG might alternately induce apoptotic pathways in LKB1-null cell lines, reducing cell number. To test this hypothesis, we compared apoptosis-related transcripts of $\mathrm{H} 23$ (LKB1-null) and H2009 (LKB1-positive) cells by treatment with either low-dose $(2.5 \mathrm{mmol} / \mathrm{L})$ or high-dose $(20 \mathrm{mmol} /$ L) 2-DG or vehicle (phosphate-buffered saline) for 6 hours followed by microarray-based assessment of gene expression. Expression analysis revealed dramatic changes in 


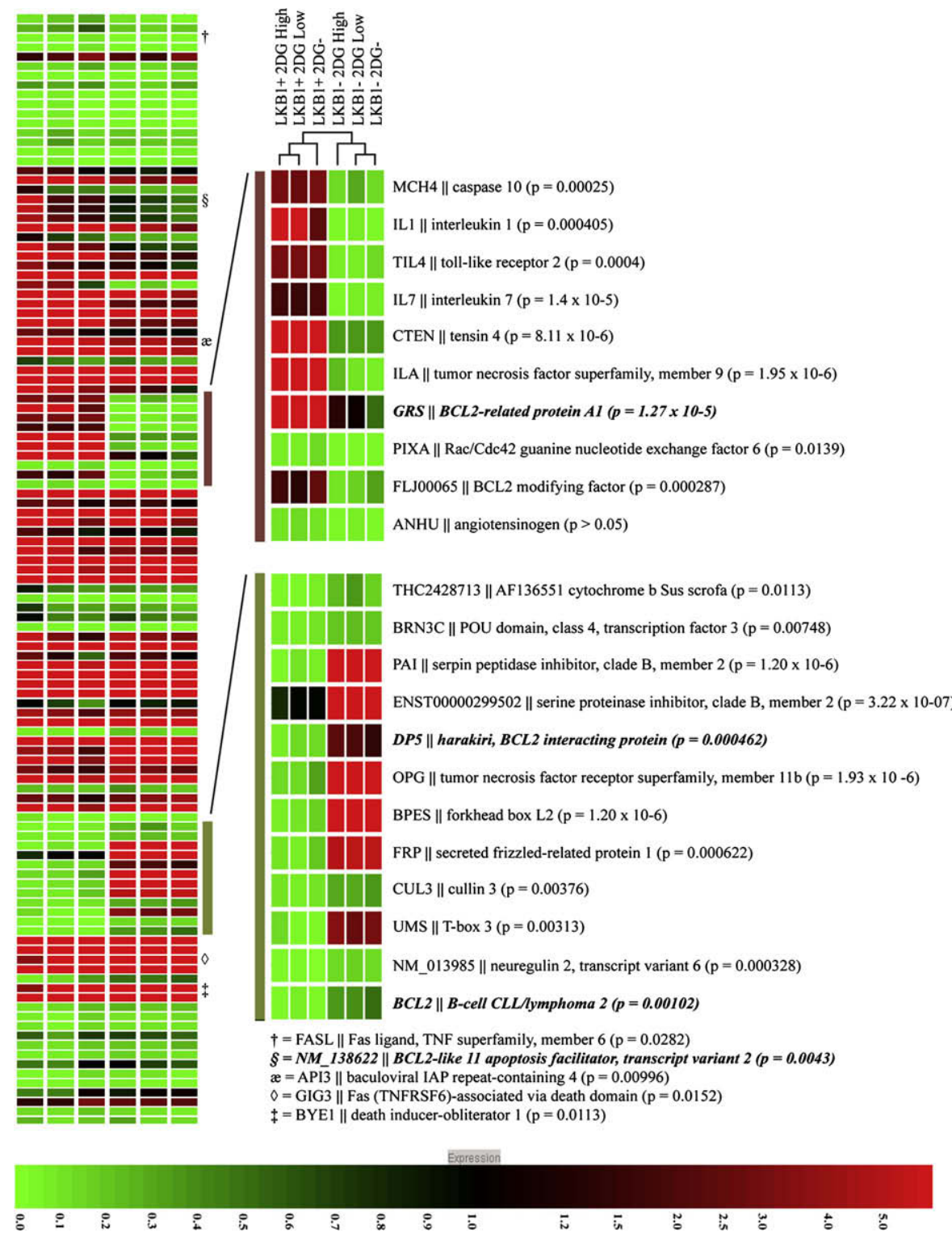

FIGURE 3. Microarray analysis of LKB1-positive (LKB1+) or LKB1-negative (LKB1-) NSCLC cell lines (H2009 and H23, respectively) treated or untreated, in a dose-dependent manner, with 2-DG. Unsupervised hierarchical clustering was performed on 117 apoptosis-related transcripts (left). Excerpted gene clusters are shown at the right. The pattern of gene expression reflects an activation of apoptosis in LKB1-negative cells. Italicized, bolded genes are discussed in the text. 2-DG, 2-Deoxyglucose.

a variety of genes owing to 2-DG treatments in both $\mathrm{H} 2009$ and H23 (Figure 3). Interestingly, although we found little difference in expression of the pro-apoptotic protein, Fas ligand, and its related proteins, we observed dose-dependent changes in the expression of the anti-apoptotic protein, BCL-2, and several BCL-2 interacting proteins in LKB1null $\mathrm{H} 23$ cells (Figure 3 ). Taken together, these results suggested an activation of apoptosis within H23 LKB1-null NSCLC cells, but not H2009 cells, by 2-DG. To confirm that 2-DG activated apoptosis in LKB1-null, but not LKB1-positive NSCLC cells, H23 and H2009 were treated with increasing doses of 2-DG for 24 hours and assayed for activation of caspases 3 and 7. Treatment with 2-DG induced a dose-dependent activation of caspases 3 and 7 in $\mathrm{H} 23$ cells, consistent with the gene expression data (Figure $4, A$ ). Further, immunoblot analysis of cleaved PARP, a marker of apoptosis, was present in 2-DG-treated $\mathrm{H} 23$ cells, but not in $\mathrm{H} 2009$ cells (Figure 4, B). Thus, these findings demonstrate that 2-DG induces apoptosis in LKB1null but not in LKB1-positive cells.

To understand whether the lack of LKB1 function was responsible for the effects of 2-DG on NSCLC cells, we re-introduced a FLAG-tagged LKB1 into $\mathrm{H} 23$ cells via retroviral infection. In addition, an inactive, kinase-dead form 

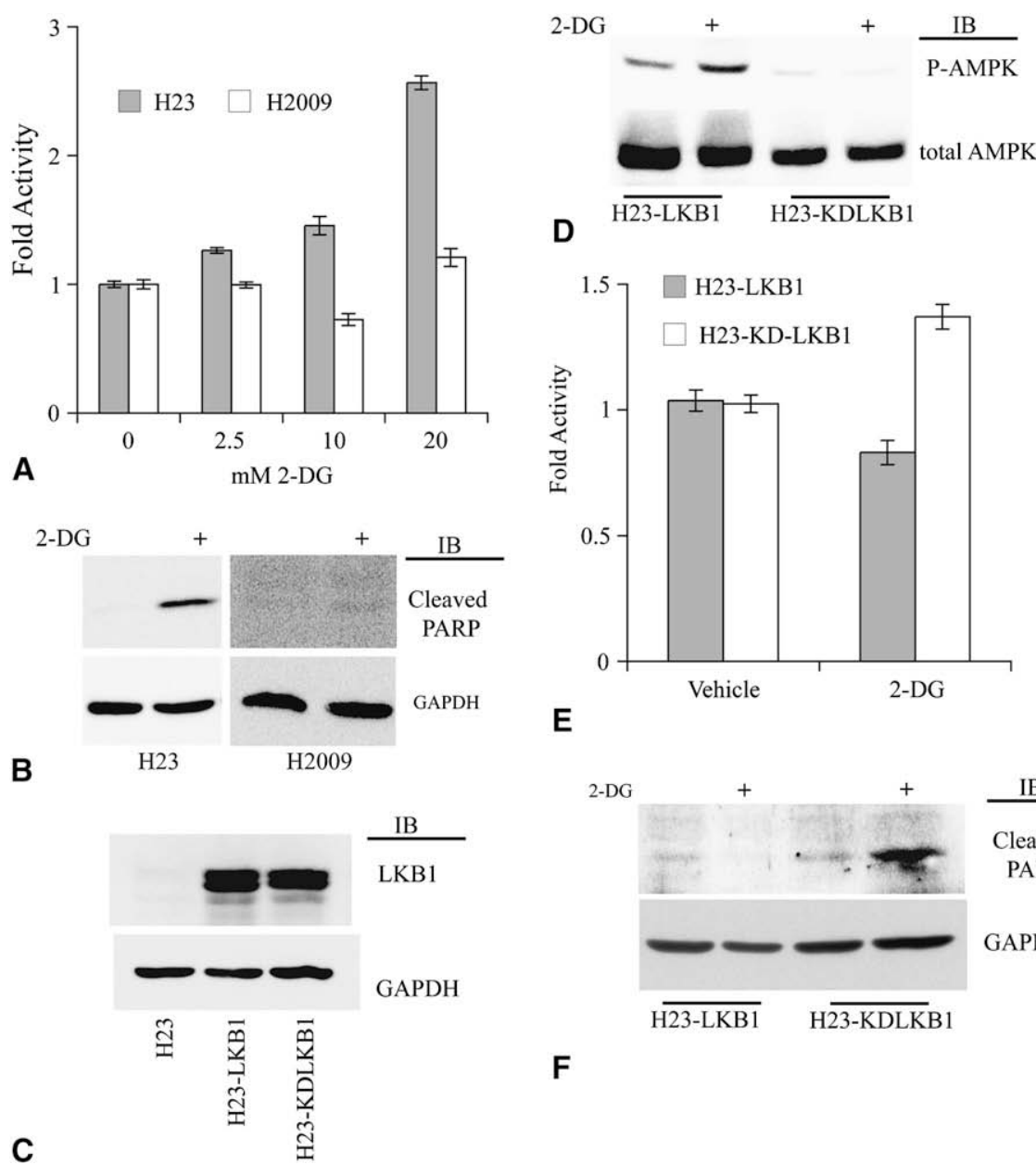

E

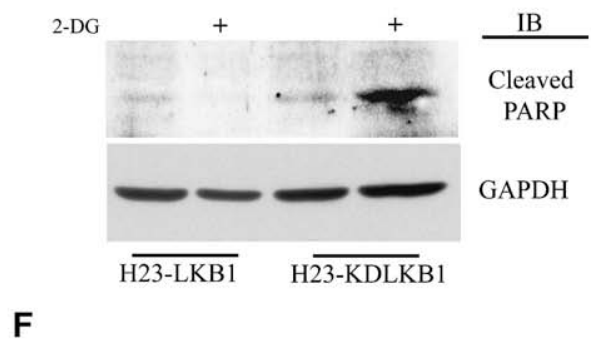

FIGURE 4. 2-DG induces apoptosis in LKB1-null NSCLC cells. A, Activity of caspases 3 and 7 in NSCLC cells after 24 hours of 2-DG treatment at the indicated concentrations. Bars represent standard error and experiment was repeated twice. B, Immunoblot of cleaved PARP. H23 and H2009 cells were treated for 24 hours with $20 \mathrm{mmol} / \mathrm{L}$ of 2-DG. Note presence of cleaved PARP in H23, but not H2009 cells. GAPDH was used as a loading control. C, Immunoblot of LKB1 and KDLKB1 in H23 cells after retroviral infection and puromycin selection. GAPDH was used as a loading control. D, 2-DG activation of LKB1 in H23-LKB1, but not H23-KDLKB1 cells. Blot was stripped and probed for AMPK as a loading control. Blot represents two independent experiments. E, LKB1, but not KDLKB1, prevents activation of capase 3 and 7 in H23 cells. H23-LKB1 and H23-KDLKB1 cells were treated for 24 hours with $20 \mathrm{mmol} / \mathrm{L}$ 2-DG before analysis as described in Materials and Methods. Bars represent standard error and experiment was repeated twice. F, Immunoblot of cleaved PARP in H23-LKB1 and H23-KDLKB1 after treatment with $20 \mathrm{mmol} / \mathrm{L}$ of 2-DG for 24 hours. Blot is representative of two independent experiments. GAPDH was used as a loading control. 2-DG, 2-Deoxyglucose; $P A R P$, poly (ADP-ribose) polymerase; $A M P K$, adenosine monophosphate activated protein kinase; $K D-L K B 1$, kinase-dead LKB1.

of LKB1 (KDLKB1) was also introduced using the same retroviral vector as a negative control. $\mathrm{H} 23$ cells were infected with either FLAG-tagged LKB1 or KDLKB1 retroviruses and underwent puromycin selection. The resulting cell lines, H23-LKB1 and H23-KDLKB1, both express LKB1, as shown by Western blot (Figure 4,C). However, only H23-LKB1 is capable of phosphorylating AMPK when treated with 2-DG, whereas H23-KDLKB1 does not (Figure $4, D$ ). Re-expression of LKB1 in $\mathrm{H} 23$ cells prevented 2-DG-induced activation of caspase 3 and 7, but not in H23 cells expressing KDLKB1 (Figure 4, E). In addition, re-expression of LKB1 in $\mathrm{H} 23$ cells also prevented cleavage of PARP after treatment with 2-DG, but not in $\mathrm{H} 23$
KDLKB1 cells (Figure 4, F). In sum, these findings demonstrate that LKB1 function is critical in determining the effects of 2-DG in NSCLC cells.

\section{DISCUSSION}

Directing therapy based on the unique characteristics of each individual's cancer (personalized therapy) has many potential benefits. The recent identification of unique EGFR mutations in some NSCLCs has led to the more effective use of EGFR inhibitors. ${ }^{2}$ It is clear that biological approaches to lung cancer have the potential to affect the overall survival from this disease, which historically has lagged behind the success of treatment for cancers of other 
organ sites. Similarly, understanding molecular characteristics of a cancer has led to attempts at improving the outcomes of traditional chemotherapeutic agents by directing these agents to those cancers most likely to respond. ${ }^{19,20}$ Currently, the molecular diversity of NSCLC and the complexity of its response to traditional agents suggest that success in improving the outcomes for this disease will rely in part on individualized therapy. To this end, we have attempted to exploit the frequent inactivation of LKB1 in NSCLC to design a potential therapy for patients with these tumors. LKB1 appears to be inactivated/mutated in up to $50 \%$ of NSCLC ${ }^{8}$ representing a large group of patients who could benefit from this therapy. Inasmuch as LKB1 is associated with the regulation of cellular metabolism, we hypothesized that a glycolytic inhibitor such as 2-DG could interfere with the survival of cells with inactive LKB1.

We found that 2-DG was a potent activator of apoptosis in LKB1-null cells, which confirms previous studies that have demonstrated activation of apoptosis after induction of energetic stress in LKB1-null cells. ${ }^{12-14}$ The 2-DG-dependent activation of apoptosis was prevented by re-expression of LKB1 in LKB1-null cells, highlighting the critical role of LKB1 in metabolic adaptation. During conditions of reduced nutrient availability, cells enter into either autophagy, a process in which energy is derived via catabolism of intracellular organelles, or apoptosis. ${ }^{21,22}$ LKB1 activity appears to play a role in this decision, as stabilization of the cell cycle inhibitor, p27/kip1, by LKB1 results in autophagy, instead of apoptosis during energetic stress. ${ }^{23}$ Thus, 2-DG treatment may result in autophagy in LKB1-expressing NSCLC cells, an area we are currently investigating. However, other regulatory functions of LKB1 cannot be discounted inasmuch as inhibition of mTOR with rapamycin can also prevent cell death during energetic stress in LKB1-null cells, ${ }^{12,14}$ while we have observed increased mTOR activation in LKB1null cells treated with 2-DG (unpublished observations). These findings suggest that the inability to regulate mTOR activity in LKB1-null cells during treatment with 2-DG may result in apoptosis owing to undetermined mechanisms. We are currently investigating these and other possible mechanisms for 2-DG toxicity in LKB1-null NSCLC cells.

Gene expression analysis revealed dose-dependent changes in BCL-2 and BCL-2-related genes in LKB1null cells, as well as activation of caspases and caspase function (see Figures 3 and 4), suggesting that LKB1-null cells undergo apoptosis via a mitochondrion-mediated mechanism. Surprisingly, we also observed changes in apoptotic and inflammatory genes in LKB1-expressing cells with 2-DG treatment (specifically, interleukin 1; see Figure 3). The increases in these genes suggest that although these cells did not undergo apoptosis in our in vitro system, it is possible that in vivo, the expression of these genes may induce recruitment of inflammatory cells, which might activate apoptosis through receptor-mediated pathways. Alter- natively, cells in extended periods of autophagy undergo cell death via nonapoptotic type II pathway, ${ }^{21,22}$ which cannot be determined by our experimental design and could generate our observed decreases in cell viability in LKB1expressing NSCLC after 2-DG treatment (Figure 2,C). Thus, it is of critical importance to extend these studies into in vivo models, as well as studying the effects of 2DG on LKB1-expressing NSCLC cells, areas that we are actively pursuing.

2-DG is currently undergoing phase I trials in combination with standard chemotherapeutics for the treatment of various solid tumors. Our work suggests that categorizing patients on the basis of LKB1 expression will yield improved patient response to 2-DG. This treatment paradigm is similar to the use of EGFR inhibitor, Tarceva, in NSCLC patients with distinct mutations within EGFR, which occur in only $10 \%$ to $15 \%$ of patients with NSCLC. ${ }^{2}$ Current statistics have suggested LKB1 loss may occur in $30 \%$ to $50 \%$ of NSCLC. However, NSCLC is a heterogeneous disease consisting of large cell carcinoma, adenocarcinoma, squamous cell carcinoma, and mixed histology tumors (adenosquamous). Among these subtypes, LKB1 loss appears to occur most frequently in adenocarcinoma (34\%), with LKB1 loss occurring in squamous cell carcinoma (19\%), large cell carcinoma (14\%), and adenosquamous $(25 \%)$ at lower rates. ${ }^{8}$ On the basis of these findings, we initially chose to focus our studies on adenocarcinoma cell lines. However, it is plausible that 2-DG would induce a similar response in squamous cell, large cell, and adenosquamous tumors, a subject currently under investigation in our laboratory. Despite the variances of LKB1 loss within the NSCLC subtypes, 2-DG would benefit a substantially larger patient population than would Tarceva-sensitive NSCLC. Owing to the significant lack of treatment options available to patients with NSCLC, we believe that the targeting of metabolic processes within LKB1-null NSCLC tumor will provide a new avenue for treatment in this disease.

\section{References}

1. Jemal A, Siegel R, Ward E, Murray T, Xu J, Thun MJ. Cancer statistics, 2007. CA Cancer J Clin. 2007;57:43-66.

2. Sharma SV, Bell DW, Settleman J, Haber DA. Epidermal growth factor receptor mutations in lung cancer. Nat Rev. 2007;7:169-81.

3. Alessi DR, Sakamoto K, Bayascas JR. LKB1-dependent signaling pathways. Ann Rev Biochem. 2006;75:137-63.

4. Matsumoto S, Iwakawa R, Takahashi K, Kohno T, Nakanishi Y, Matsuno Y, et al. Prevalence and specificity of LKB1 genetic alterations in lung cancers. Oncogene. 2007;26:5911-8.

5. Sanchez-Cespedes M, Parrella P, Esteller M, Nomoto S, Trink B, Engles JM, et al Inactivation of LKB1/STK11 is a common event in adenocarcinomas of the lung. Cancer Res. 2002;62:3659-62.

6. Ji H, Ramsey MR, Hayes DN, Fan C, McNamara K, Kozlowski P, et al. LKB1 modulates lung cancer differentiation and metastasis. Nature. 200716;448 807-10.

7. Sanchez-Cespedes M. A role for LKB1 gene in human cancer beyond the PeutzJeghers syndrome. Oncogene. 2007;26:7825-32.

8. Shah U, Sharpless NE, Hayes DN. LKB1 and lung cancer: more than the usual suspects. Cancer Res. 2008;68:3562-5. 
9. Shaw RJ. Glucose metabolism and cancer. Current Opin Cell Biol. 2006;18: 598-608.

10. Inoki K, Zhu T, Guan KL. TSC2 mediates cellular energy response to control cell growth and survival. Cell. 2003;115:577-90.

11. Guertin DA, Sabatini DM. Defining the role of mTOR in cancer. Cancer Cell. 2007;12:9-22.

12. Corradetti MN, Inoki K, Bardeesy N, DePinho RA, Guan KL. Regulation of the TSC pathway by LKB1: evidence of a molecular link between tuberous sclerosis complex and Peutz-Jeghers syndrome. Genes Dev. 2004;18:1533-8.

13. Carretero J, Medina PP, Blanco R, Smit L, Tang M, Roncador G, et al. Dysfunctional AMPK activity, signalling through mTOR and survival in response to energetic stress in LKB1-deficient lung cancer. Oncogene. 2007;26:1616-25.

14. Shaw RJ, Bardeesy N, Manning BD, Lopez L, Kosmatka M, DePinho RA, et al. The LKB1 tumor suppressor negatively regulates mTOR signaling. Cancer Cell. 2004;6:91-9.

15. Karczmar GS, Arbeit JM, Toy BJ, Speder A, Weiner MW. Selective depletion of tumor ATP by 2-deoxyglucose and insulin, detected by 31P magnetic resonance spectroscopy. Cancer Res. 1992;52:71-6.

16. Maschek G, Savaraj N, Priebe W, Braunschweiger P, Hamilton K, Tidmarsh GF, et al. 2-Deoxy-d-glucose increases the efficacy of adriamycin and paclitaxel in human osteosarcoma and non-small cell lung cancers in vivo. Cancer Res. 2004;64:31-4.
17. Simons AL, Ahmad IM, Mattson DM, Dornfeld KJ, Spitz DR. 2-Deoxy-d-glucose combined with cisplatin enhances cytotoxicity via metabolic oxidative stress in human head and neck cancer cells. Cancer Res. 2007;67:3364-70.

18. Garriga-Canut M, Schoenike B, Qazi R, Bergendahl K, Daley TJ, Pfender RM, et al. 2-Deoxy-d-glucose reduces epilepsy progression by NRSF-CtBP-dependent metabolic regulation of chromatin structure. Nat Neurosci. 2006;9:1382-7.

19. Ceppi P, Volante M, Novello S, Rapa I, Danenberg KD, Danenberg PV, et al. ERCC1 and RRM1 gene expressions but not EGFR are predictive of shorter survival in advanced non-small-cell lung cancer treated with cisplatin and gemcitabine. Ann Oncol. 2006;17:1818-25.

20. Lord RV, Brabender J, Gandara D, Alberola V, Camps C, Domine M, et al. Low ERCC1 expression correlates with prolonged survival after cisplatin plus gemcitabine chemotherapy in non-small cell lung cancer. Clin Cancer Res. 2002;8: 2286-91.

21. Lum JJ, DeBerardinis RJ, Thompson CB. Autophagy in metazoans: cell survival in the land of plenty. Nat Rev Mol Cell Biol. 2005;6:439-48.

22. Reggiori F, Klionsky DJ. Autophagosomes: biogenesis from scratch? Curr Opin Cell Biol. 2005; 17:415-22.

23. Liang J, Shao SH, Xu ZX, Hennessy B, Ding Z, Larrea M, et al. The energy sensing LKB1-AMPK pathway regulates p27(kip1) phosphorylation mediating the decision to enter autophagy or apoptosis. Nat Cell Biol. 2007;9:218-24. 